\title{
Incidencia del Aprendizaje Basado en Proyectos, implementado con Tecnologías de Información y Comunicación, en la motivación académica de estudiantes de secundaria*
}

\author{
Impact of the didactic strategy Project-Based Learning, \\ implemented with Information and Communication \\ Technologies, in the academic motivation of high school \\ students*
}

Impacto da estratégia didática Aprendizagem Baseada em Projetos, implementada com Tecnologias de Informação e Comunicação, na motivação acadêmica de estudantes do ensino médio. *

Ricardo Luciano Chaparro Aranguren **

Johanna Natalia Barbosa Sánchez ***

\section{Universidad Manuela Beltrán - Colombia Universidad de la Sabana}

Fecha de Recibido: Febrero 10 del 2018

Fecha de Aceptación: Julio 26 de 2018

Fecha de Publicación: Agosto 01 de 2018

DOI: http://dx.doi.org/10.22335/rlct.v10i4.647

*El artículo resultado de la investigación desarrollada por la Universidad Manuela Beltrán Año 2018 en la Institución Educativa San Sebastián Colombia, Huila

**Magister en Informática Educativa. Investigador titular en la Maestría en Tecnologías Digitales Aplicadas a la Educación UMB, Universidad de la Sabana, Filiación: Universidad Manuela Beltran. Colombia. Correo electronico: ricardo.chaparro@umb.edu.co Orcid https://orcid.org/0000-0002-5157-6910

*** Magister en Tecnologías Digitales Aplicadas a la Educación. Filiación: Institución Educativa San Sebastián Colombia, Huila Correo electrónico: johannabarbosa.ns@academia.umb.edu.co Orcid: https://orcid.org/0000-0002-0069-6752

\section{Resumen}

La investigación está destinada a identificar la incidencia de un ambiente de Aprendizaje Basado en Proyectos (ABP) mediado con TIC, en la motivación académica de estudiantes de secundaria en la enseñanza de conceptos básicos de circuitos eléctricos, en la temática de corriente eléctrica del área de tecnología. La unidad de análisis fue el grado 9, cuyas edades oscilan entre 14 y 16 años de edad. Se utilizó enfoque cualitativo con diseño de estudio de caso y técnicas de grupo focal y observación. El análisis fue la teoría fundamentada evidenciando una contribución significativa en el interés de los estudiantes conocido como "interés situacional", surgiendo, respuestas atencionales y de implicación académica que ayudan a orientar la conducta hacia una motivación académica. 
Palabras clave: Motivación Académica, Interés situacional, ABP, TIC, Tecnología.

\begin{abstract}
The research is aimed at identifying the impact of an environment of Project-Based Learning ( $\mathrm{PBL}$ ) mediated with ICT, on the academic motivation of high school students in the teaching of basic concepts of electrical circuits, in the subject of electric current in the area of technology. The unit of analysis was grade 9 , whose ages range between 14 and 16 years of age. A qualitative approach was used with case study design and focus group and observation techniques. The analysis was grounded theory evidencing a significant contribution in the interest of students known as "situational interest", arising, attentional responses and academic involvement that help guide behavior toward academic motivation.
\end{abstract}

\section{Keywords:}

Academic Motivation, Situational Interest, PBL, ICT, Technology.

\section{Resumo}

A pesquisa tem como objetivo identificar o impacto de um ambiente de Aprendizagem Baseada em Projetos (ABP) mediado com as TIC, sobre a motivação acadêmica de estudantes do ensino médio no ensino de conceitos básicos de circuitos elétricos, no tema corrente elétrica na área de tecnologia A unidade de análise foi a $9^{a}$ série, com idades entre 14 e 16 anos. Utilizou-se abordagem qualitativa com delineamento de estudo de caso e grupo focal e técnicas de observação. A análise foi fundamentada na teoria evidenciando uma contribuição significativa no interesse dos alunos, conhecida como "interesse situacional", surgindo, respostas atencionais e envolvimento acadêmico que ajudam a orientar o comportamento em direção à motivação acadêmica.

Palavras-chave: Motivação Acadêmica, Interesse Situacional, PBL, TIC, Tecnologia.

\section{Introducción}

Con el propósito de dar un aporte a la relación manifiesta entre el tema del aprendizaje y la motivación que presentan los estudiantes en las aulas de clase, y entendiendo que para aprender algo nuevo es necesario no solo disponer de capacidades, destrezas y conocimientos "poder", sino además, tener la disposición, intensión o interés suficientes "querer" (Núñez, 2009), llama la atención datos y publicaciones de la UNESCO, OCDE, investigaciones durante los últimos 4 años, que hacen evidente la percepción de conformidad y de indiferencia de los estudiantes en el contexto escolar, convertida en una problemática inquietante para padres, docentes y Ministerio de Educación Nacional (MEN), que ha llevado a proponer el trabajo de la motivación a través de la curiosidad científica y tecnológica, con la publicación de las Orientaciones Generales para Educación en Tecnología las cuales "pretenden motivar a niños, niñas, jóvenes y maestros hacia la comprensión y la apropiación de la tecnología" (MEN, 2008:3).

El abordaje de la motivación académica en el aula de clase y la búsqueda continua de estrategias metodológicas que permiten el aprendizaje en los estudiantes, es uno de los grandes temas de investigaciones en todo el mundo. Lo anterior, al reconocer como factor inherente al ser humano y determinante de la conducta que promueve los procesos de aprendizaje.

En Colombia, una investigación realizada en la Universidad de la Sabana titulada. Las TICS como herramienta de motivación en el aula (Ospina, 2013), presenta los resultados de un proyecto de investigación que explora el potencial motivacional de las TIC en el marco del proceso de formación de estudiantes de básica secundaria y media, en la asignatura de Tecnología e Informática, en una institución educativa rural de carácter oficial.

El proyecto estableció su experiencia investigativa de naturaleza cualitativa durante un año y medio, permitiendo a través de la observación directa con los estudiantes, un análisis de trabajos presentados, encuestas y aplicación de entrevistas; poder identificar la relación entre los factores asociados a la motivación y a la integración educativa de las TIC. 
Por otra parte, se generó un escenario de investigación colectivista más que individualista, el cual se centró en la pluralidad, variedad de las actitudes, experiencias y vivencias de los participantes. Lo que permitió profundizar en las experiencias y percepciones que tuvieron los educandos que participaron.

Los resultados obtenidos en la investigación, reflejan que el factor de atribución causal, como esfuerzo, capacidad, dominio del tema, habilidades, representó que la práctica dentro y fuera de la clase, son un factor fundamental en el aumento de la motivación. Por otra parte, el factor denominado, Auto Concepto (responsabilidad, compromiso e interés, creatividad, presentación, seguimiento de instrucciones) también fue significativo al presentar un índice de disposición para estar involucrado en las actividades propuestas; se reconoció de parte de los educandos un gusto por la actividad y comprendieron su utilidad, mejoraron el valor dela responsabilidad, lo que implico constancia y esfuerzo, dirigiendo estos intereses hacia la obtención del logro.

En consecuencia, el aporte para la investigación fue permitir conocer factores determinantes para el estudio de la motivación académica, igualmente es de analizar que las TIC son herramientas a tener en cuenta para despertar motivación que debe estar complementada con estrategias metodológicas definidas por el docente en su plan curricular.

Por otra parte, Domínguez, Carod \& Velilla (2008), sugieren cuestionar los métodos de aprendizaje y reflexionar sobre la forma más adecuada para que un estudiante adquiera la capacidad de aprendizaje continuo, en el que muchos de los estudiantes, buscan apropiarse del conocimiento científico mediante la memorización de ecuaciones y definiciones. Otros realizan análisis de problemas científicos con estrategias de razonamiento y metodologías superficiales. Lo anterior, hacen énfasis en que una enseñanza basada únicamente en la transmisión de conocimientos es claramente insuficiente. (p. 1-6)

En este sentido, se ha indagado experiencias de aprendizaje de manera significativa, donde se encuentran diversos estudios con enfoques que plantean vencer las dificultades del proceso de aprendizaje. Es de mencionar, Aprendizaje Basado en Problemas ABP, Aprendizaje Orientado a Proyectos. Para la investigación se entiende como ABP.

Es el caso de una investigación realizada en Colombia en la Universidad Nacional, aplicada a estudiantes del grado undécimo del Colegio Santa María del Rosario en la ciudad de Medellín (Aristizabal, 2012), cuyo objetivo consiste en recopilar lineamientos básicos para la implementación de la metodología de $\mathrm{ABP}$, como estrategia integradora de teoría y práctica para cualquier área o asignatura, buscando promover competencias cognitivas, colaborativas, tecnológicas y metas cognitivas. El estudio permitió comprender en el contexto real, la aplicación de conceptos y leyes de la física mecánica a través del diseño, articulando conocimientos propios de la disciplina de forma crítica y analítica.

Según los resultados de la investigación, la aplicación de estrategias por proyectos significó una ruptura con la metodología tradicional de enseñanza, desarrollando cambios de actitud del docente, la institución y estudiantes. Igualmente, fortaleció el trabajo cooperativo y colaborativo en el desarrollo de diversas fases del proyecto; donde los estudiantes se mostraron proactivos al interactuar con las actividades. Es de mencionar que la investigación, se consolidaron relaciones del docente con los estudiantes, favoreciendo la discusión y desarrollando un ambiente de confianza. Lo anterior, para incentivar la participación con aportes e inquietudes.

Un factor determinante en las investigaciones que se encontraron a nivel global, nacional, que aportaron conceptos y metodologías en los procesos del artículo, argumentan que la motivación de los estudiantes en el aula es muy importante, preocupando a psicólogos y a docentes, por considerarse un factor determinante de los resultados académicos.

Es entonces que en el intento por comprender aspectos motivacionales de los estudiantes en un proceso de aprendizaje, y dado que la motivación es un constructo con diferentes componentes, se 
encuentran variados estudios en el que se evalúan aspectos como: características personales de los alumnos que afectan a su esfuerzo por aprender (metas, intereses, expectativas, volición y estilos de autorregulación), características del entorno de aprendizaje (pautas docentes con implicaciones motivacionales, clima de Clase) y las características de los profesores.

Tal es el caso de un estudio realizado por las Universidades de Vigo -España y Nacional de Rio Cuarto -Argentina (González \& Paoloni, 2015), que analizó la motivación del aula a partir del interés situacional y personal hacia la Física, estableciendo en qué medida ambos resultan afectados por dos estrategias docentes, ofreciendo posibilidades de elección en clase y determinando el grado en que estrategias e interés influyen sobre la implicación, la desafección y el rendimiento.

En dicha investigación participaron 430 estudiantes, que cursaban $2^{\circ}$ de Bachillerato de la especialidad científico-tecnológica en centros de secundaria de una Comunidad Autónoma del noroeste de España. Se seleccionaron aleatoriamente 23 centros, 16 públicos y 7 privados concertados. En cada centro, los estudiantes se seleccionaron a través de muestreo por conglomerados. Los instrumentos de recolección de información fueron cuestionarios aplicando sub escalas de otros estudios, y los resultados arrojaron que el interés personal y el situacional, se vieron potenciados por estas dos estrategias docentes; además, ambos tipos de interés y las estrategias favorecieron la implicación y el rendimiento académico, protegiendo a los alumnos frente a la desafección.

Por consiguiente, es de mencionar que la problemática del artículo de investigación se desarrolló en la institución educativa San Sebastián en el departamento del Huila en Colombia, se venía percibiendo que los jóvenes presentaban falta de atención, distracción con dispositivos móviles, retrasos en las entregas y apropiación de conceptos del área de tecnología; especialmente en la enseñanza de conceptos básicos de circuitos eléctricos. Por lo anterior, se diseñó una encuesta diagnóstica aplicada a diez docentes de tecnología y 69 estudiantes. Dentro del instrumentos se evidencia un $34,78 \%$ de los estudiantes poco les interesa la asignatura de tecnología, 58,99\% se siente en desacuerdo respecto a cuanto menos contenido de tecnología e informática se brinde en clase es mejor.

Igualmente, en la encuesta se destaca que un $44,93 \%$ estudian por satisfacer a sus a sus padres, $48,57 \%$ lo hacen por una nota y poco por aprender y un $66,67 \%$ afirma que lo importante es terminar cuanto antes las actividades para hacer lo que les gusta y admiten que se pierde el interés por la estrategia del profesor.

Lo anterior, lleva a proponer la implementación de un ambiente de Aprendizaje Basado en Proyectos (ABP) mediado con herramientas TIC, utilizando herramientas interactivas como el weblog y los simuladores para la enseñanza de los conceptos básicos de circuitos eléctricos, en la unidad temática de corriente eléctrica desde el área de tecnología.

La estrategia propuesta pretende situar a los estudiantes en el centro del proceso de aprendizaje, sugiriendo un ambiente más motivador por el intercambio de ideas, la colaboración, creatividad y puesta en práctica de los conocimientos en el desarrollo de un producto final que apoyado con recursos TIC, buscando dar respuesta a la problemática de la motivación y el aprendizaje, además de responder a los fundamentos epistemológicos y pedagógicos propuestos por el Proyecto Educativo Institucional (PEI) que se aplican en la institución donde se realizó la Investigación, el cual pretende "invocar en el educando la necesidad de unir teoría y práctica, motivado por el estudio y búsqueda de soluciones de problemas a partir de la realidad objetiva y con aplicación de teorías y principios universales. Dinamizar los principios de la escuela activa en la cual el maestro debe ser un orientador y el educando un protagonista". (Institución Educativa San Sebastián, 2015:22).

De acuerdo a lo anterior, y en el intento por conocer la incidencia de la estrategia metodológica ABP con mediación de las TIC en la motivación de los estudiantes en el aula, se analiza la experiencia de aprendizaje teniendo en cuenta el interés que estos manifiestan desde las dimensiones de "interés 
situacional" e "interés personal" con un enfoque cualitativo.

Es preciso referirse en primera instancia a un aspecto central, "La motivación", como un concepto fundamental del que se viene ocupando la psicología desde hace mucho tiempo, y considerada como la fuerza activadora de la conducta humana, que asociada al aula de clases se ha denominado "motivación académica". Y aunque puede estudiarse desde variados ámbitos y teorías, el trabajo se ha centrado en el estudio de la motivación desde la perspectiva del Interés en los estudiantes (figura 1).

Desde el punto de vista etimológico, la palabra motivación está compuesta por el latín Motivus (movimiento) y el sufijo -ción (acción y efecto), entendiéndose como "un conjunto de procesos implicados en la activación, dirección y persistencia de la conducta" (Núñez, 2009:3), donde las emociones juegan un papel importante ya que "lo que nos motiva es lo que nos genera emociones y lo que nos emociona nos provoca motivación" (Fros, 2013:p.2), considerándolas ambas como activadoras de la conducta. Es entonces que la motivación académica se ha de entender como ese conjunto de procesos implicados en la activación y dirección de la conducta hacia el aprendizaje escolar.

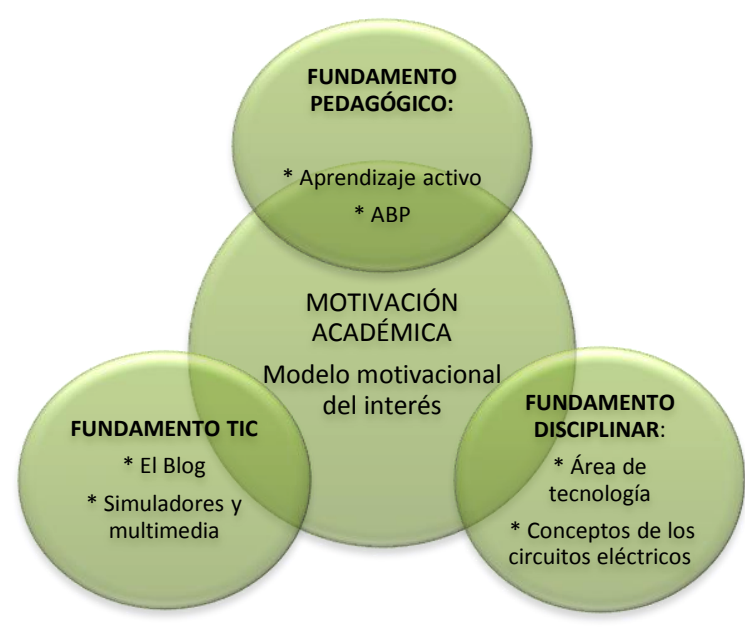

Figura 1: Esquema teórico. Fuente: Autores
Por otra parte, si algún concepto motivacional puede considerarse definitorio de la identidad propia de un estudiante, ese es su interés por un tema o una materia (Renninger, 2009), y en este sentido el interés se trata de un fenómeno que surge a partir de la interacción entre un individuo y su medio, en el que varios investigadores coinciden distinguiendo el "interés personal" y el "interés situacional."

Se define el interés personal como "aquel interés basado en el conocimiento o la valoración de una clase de objetos o ideas, que lleva al alumno a desear implicarse en actividades relacionadas con el tema" (Boekaerts y Boscolo, 2002:378), y en el interés situacional, se analizan aquellos aspectos relacionados con la tarea en sí, como puede ser los contenidos y todas aquellas condiciones ambientales que afectan el interés de los alumnos.

Es de mencionar, que la enseñanza se ha convertido en la actualidad en una educación enfocada en el estudiante como ser humano que actúa y que es parte fundamental del entorno social, que "pone a nuestros estudiantes al centro de su aprendizaje" (Fundación Enseñanza Chile, 2015:1) permitiendo espacios de participación e interacción entre los estudiantes y el docente. Ahora el alumno es el autor de su propio aprendizaje bajo la mediación de un docente que explora el aprendizaje previo para motivar el descubrimiento del nuevo aprendizaje que se deba dar, debe guiar a los alumnos a la autonomía en el aprendizaje y a ser competentes en una sociedad como la actual.

El Aprendizaje Basado en Proyectos (ABP) se considera como un modelo de "instrucción auténtico en el que los estudiantes planean, implementan y evalúan proyectos que tienen aplicación en el mundo real más allá del aula de clase" (Maldonado, 2008:5), en el que el aprendizaje, se produce como resultado del esfuerzo que realiza el alumno para resolver un problema o llevar a cabo un proyecto. Se considera como una excelente opción al reconocerle que en los estudiantes aumenta la motivación y compromiso con su propio aprendizaje, aprendiendo los conceptos de manera entretenida y adquiriendo aprendizajes más profundos. Sin embargo aunque es un método que puede 
desarrollarse de varias formas, se requiere la estructuración de un conjunto de etapas.

Igualmente, es necesario identificar el concepto de la Educación en Tecnología ya que el desarrollo científico y tecnológico se ha convertido en uno de los factores más sobresalientes en la sociedad contemporánea, donde la ausencia de una globalización mundial, sería imposible. Lo anterior, sin el avance de las fuerzas productivas de la ciencia y la tecnología. La alfabetización científica y tecnológica en Latinoamérica es una necesidad apremiante, al pretender que todos los individuos estén en capacidad para acceder, utilizar, evaluar y transformar artefactos, procesos y sistemas tecnológicos para la vida social y productiva, "debido a que el mundo y la sociedad en la que vivimos enfrenta grandes cambios relacionados con el desarrollo de la ciencia y la tecnología, los cuales están transformando nuestros estilos de vida" (Colorado y Rodríguez, 2014:12), sin embargo la educación en tecnología ha sido confundida con la alfabetización informática, ya que existe la creencia de que formar en tecnología es ofrecer una capacitación para manejar artefactos, tomando el computador y sus programas como sinónimo de la misma. El uso de los artefactos tecnológicos, incluso con un elevado nivel de sofisticación, no implica, necesariamente, que se posea una cultura tecnológica.

Por lo tanto, pretender proyectos educativos desde el área de tecnología, es la clave para permitir la vivencia de actividades relacionadas con la naturaleza del conocimiento tecnológico donde se integren la tecnología, el descubrimiento y el diseño, que den alcance a las competencias del MEN (2008) a través de las Orientaciones Generales para la Educación en Tecnología, puesto que éstas constituyen el eje articulador de todo el sistema educativo. Entiéndase por competencia según el MEN como aquellos conocimientos, habilidades y destrezas que desarrolla una persona para comprender, transformar y participar en el mundo en el que vive.

Luego, se propone que desde el área de tecnología se logre mantener el interés de los estudiantes a través del estímulo de la curiosidad científica y tecnológica, reconocer el saber tecnológico como solución a los problemas que transforman el entorno y desarrollar la reflexión crítica frente a las relaciones entre tecnología y sociedad.

La multitud de información, la velocidad de transmisión, la utilización de múltiples medios (textos, imagen, sonido, video) han hecho que durante los últimos 10 años se hayan presentado importantes cambios en educación que las ha convertido en "la innovación educativa del momento que permiten a los docentes y alumnos cambios determinantes en el quehacer diario del aula y en el proceso de enseñanza-aprendizaje" (Gómez y Macedo, 2010:3).

No obstante, aunque las TIC aplicadas a la enseñanza, favorezcan el aprendizaje de los alumnos, aumenten la motivación, incremente su interés y su creatividad, mejoren la capacidad para resolver problemas, potencien el trabajo en grupo, refuercen su autoestima y permitan una mayor autonomía en el aprendizaje, además de superar las barreras del tiempo y del espacio (Amar, 2006), las TIC por si solas no contribuyen a mejorar la educación si los docentes no saben cómo aprovecharlas en la orientación de sus alumnos y por el contrario las subutilizan. El alumno debe ser capaz de gestionar su propio aprendizaje frente al cúmulo de información disponible, la educación secundaria debe ser direccionada por un docente convertido ahora en un guía de los pasos de sus alumnos que propicie el autoaprendizaje y entienda que las TIC forman parte de un proceso que puede mejorar la capacidad de aprendizaje de las personas.

Adicionalmente, las TIC han y seguirán evolucionado, pues aunque empezaron conociendo como TIC, "en esta última década se han convertido en Tecnologías para el Aprendizaje y el Conocimiento (TAC), a lo largo de la vida. En esta segunda década del siglo XXI, las TIC e Internet están adquiriendo un protagonismo inusitado para llevar adelante muchas iniciativas sociales.... Y se están transformado en Tecnologías para el Empoderamiento y la Participación social (TEP)" (Departamento de Educación Universidades e investigación del gobierno Vasco, 2012: 11). 


\section{Método}

La investigación presenta un enfoque cualitativo, al analizar fenómenos de carácter social, como la motivación de los estudiantes a partir de unas estrategias metodológicas activas que se han de vivenciar en el aula. Se parte de que "su esencia se enfoca en comprender estos fenómenos explorando desde la perspectiva de los participantes en un ambiente natural y en relación con su contexto" (Hernández, 2014:358), y que la preocupación directa del investigador se concentra en las vivencias de los participantes tal como son sentidas y experimentadas para formar creencias propias sobre el fenómeno estudiado (p. 8 - 10). Es entre tanto la motivación un fenómeno abstracto que involucra el comportamiento humano y ha de ser descrito a partir de interacciones con su contexto. En este sentido, los métodos cualitativos son humanistas (Taylor y Bogdan, 2002: 21).

Se toma el diseño de Estudio de Caso con alcance descriptivo al permitir estudiar el caso de un "programa educativo innovador" (Stake, 1999: 12 16) como el ABP incorporando las TIC aplicado a estudiantes de secundaria, basado en el entendimiento comprensivo de las situaciones en el aula en torno al fenómeno motivacional de interés, para analizar y describir la incidencia de este "programa" o estrategia metodológica en la motivación académica. Este tipo de diseño es "permite el estudio directo de personas o grupos durante un cierto periodo en escenarios comunes, utiliza la observación participante para conocer la vida cotidiana del grupo desde el interior del mismo" Bisquerra (2009:310).

Se ha empleado una muestra intencional en la que la selección de la unidad de análisis estuvo sujeta a criterios a priori que llevan a elegir el grado noveno integrado por 42 participantes con edades que oscilan entre 14 y 16 años de edad, con criterios como la posibilidad de acceso al grupo, tiempo de asignación académica (Stake, 1999: p.17).

Para la recolección de los datos una de las técnicas utilizadas corresponde al grupo focal, utilizada en investigación cualitativa en todos los campos del conocimiento, cuyo objetivo es el de generar y analizar la interacción de varios participantes y como se construyen grupalmente significados. En ella existe un interés de parte del investigador por como los individuos forman un esquema o perspectiva de un problema a través de la interacción (Hernández, 2014). Dicha técnica aplicada antes y durante el proceso de aprendizaje, está basada en el desarrollo de una entrevista semiestructurada de manera colectiva, en el que se formulan preguntas que pretenden recoger comentarios y expresiones que permitan identificar la motivación de los estudiantes desde la perspectiva del interés en las clases de tecnología.

La técnica de observación por medio de una rejilla, es otro instrumento de tipo cualitativo, "que permite registrar los datos con más facilidad ya que el observador cuenta con tipo de plantilla o lista, en la que puede apuntar, no solo la presencia o ausencia de determinado comportamiento, si no la intensidad y frecuencia con que se produce" (Díaz, 2011:21). Dicha plantilla pudo recoger nueva información de tipo motivacional durante el desarrollo de diversas fases del ABP, centrando la investigación en el interés que evidenciaron los estudiantes por medio de su comportamiento e interacción. 


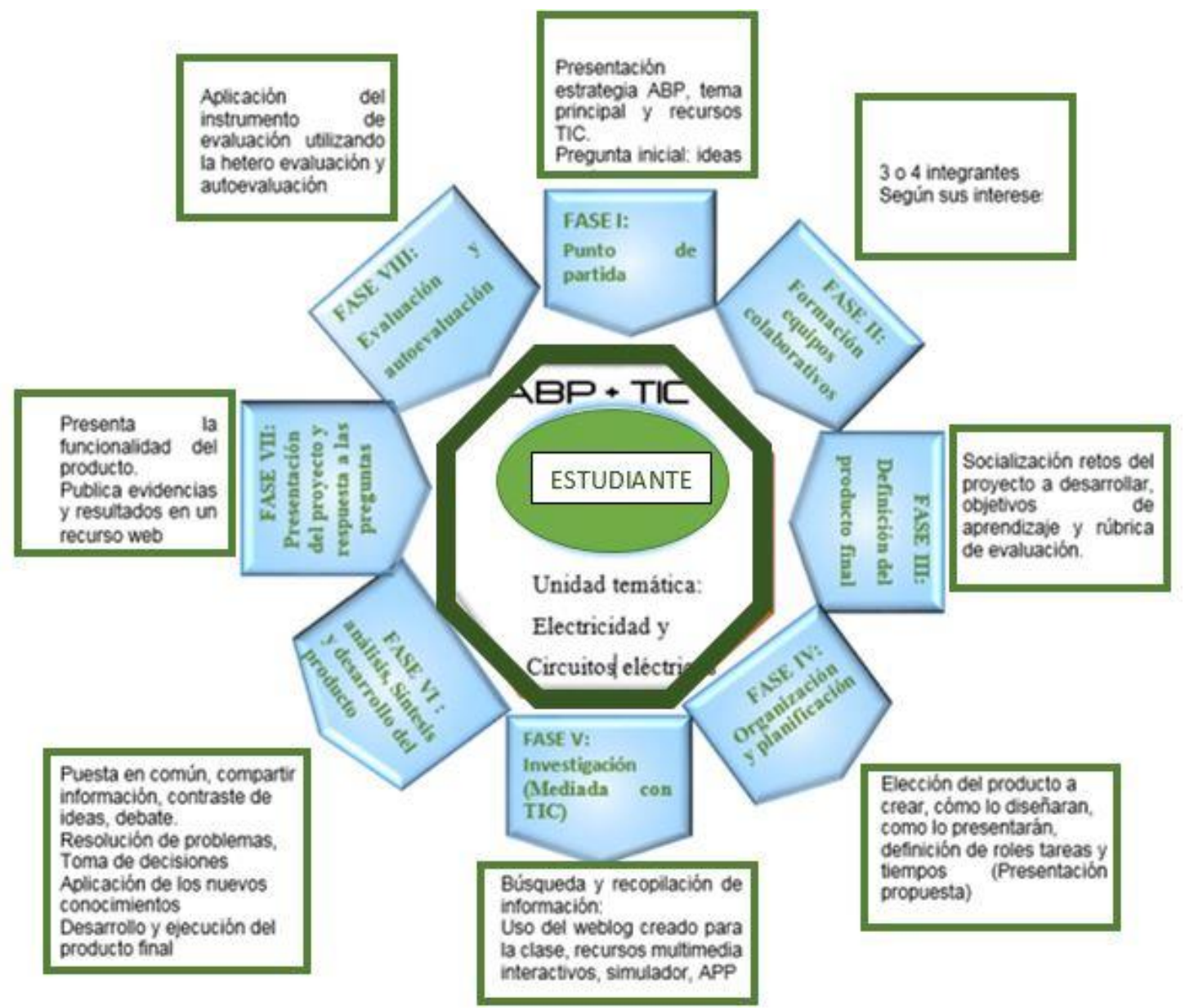

Figura 2: Representación ambiente de aprendizaje ABP con TIC aplicado durante la investigación. Fuente: Autores

Como puede observarse en la (figura 2), la investigación está enmarcada en un ambiente de aprendizaje bajo la estrategia metodológica del Aprendizaje Basado en Proyectos, con mediación de TIC, que define las condiciones $y$ circunstancias que se genera la experiencia de aprendizaje y se pretende promover un proceso progresivo y cooperativo, que motiva a los estudiantes a encontrar significados y construir conocimiento sobre conceptos y principios científicos para ser aplicados en el área de tecnología. De la misma forma es un ambiente que posibilita la interacción de los estudiantes con la realidad dentro y fuera del aula, con una perspectiva de aprender haciendo. Se puede mencionar aspectos relevantes como los sitios web para identificar y compartir conceptos de la unidad, posibilidad de descarga de documento, recursos multimedia, aplicaciones de simulación tanto para dispositivos móviles como para computadores de escritorio y portátiles, además de ejercicios que el estudiante debe estar dispuesto a desarrollar.

\section{Resultados}

Respecto al análisis de los datos en la investigación, la obtención de los resultados se logra desarrollar por medio de la teoría fundamentada, la cual "es una metodología que busca comprender el mundo expresándolo teóricamente desde la fundamentación de los datos" (Corbin y Strauss 2002), los cuales fueron recopilados de manera sistemática y analizados por medio de un proceso de investigación de 4 pasos tal como se explican en la (figura 3). De 
igual forma es de mencionar que dentro de la metodología existe un orden sistemático, donde se incluyen categorías a priori y emergentes. Gutiérrez, et.al. (2018) hace referencia que la primera fase de la teoría es una de las más importantes ya que inicia con un procedimiento analítico por medio de una Codificación abierta.

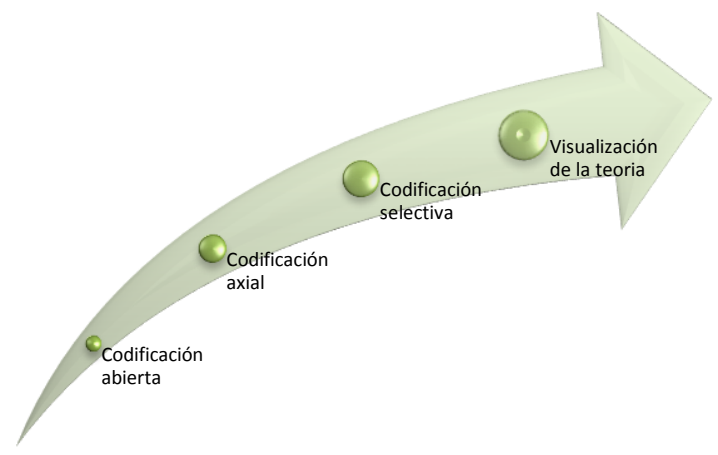

Aunque esta metodología lleva a la construcción de un marco teórico a partir del conjunto de categorías (temas y conceptos) que emergen de los datos y de la perspectiva teórica del investigador, con categorías interrelacionadas de manera sistemática explicando fenómenos sociales, psicológicos, educativos o de otra clase; conocer las teorías existentes puede ser útil, ya que es posible que el investigador se interese por extenderla llegando con conceptos y relaciones en mente, buscando propiedades y dimensiones y como varían bajo un conjunto de otras condiciones.

Teniendo en cuenta lo anterior, en la (figura 4), se presentan el conjunto de categorías y relaciones de las subcategorías, que muestran los conceptos más representativos evidenciados por los estudiantes, junto con las interpretaciones y conceptos del investigador:

Figura 3: Etapas de la teoría fundamentada aplicada en la reducción de los datos. Fuente: Autores

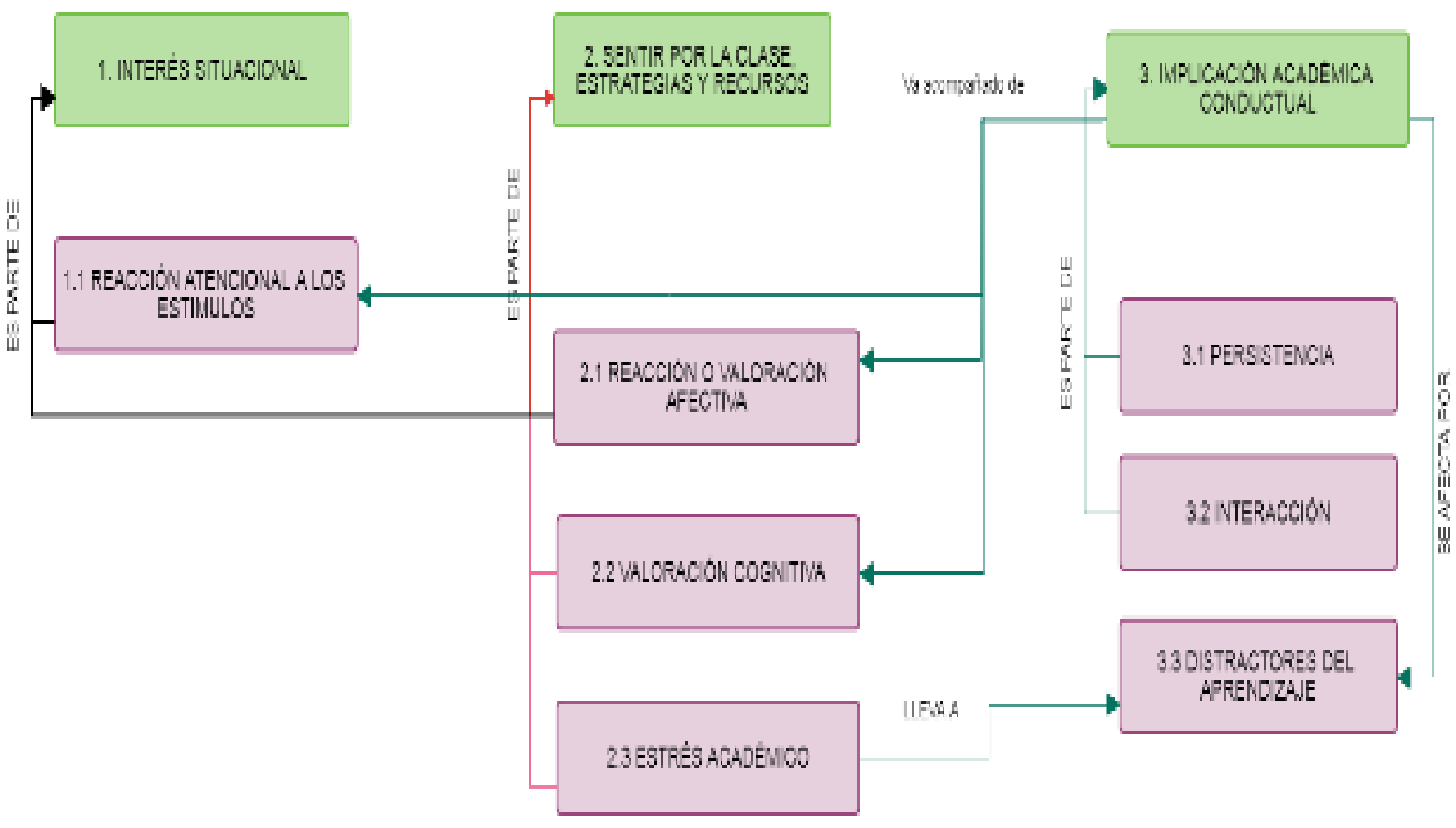

Figura 4: Diagrama de categorías obtenido como resultado del análisis. 
Respecto a la primera categoría denominada interés situacional, permite conocer en primera instancia un factor fundamental de la motivación de los estudiantes, y es qué tan interesante fue la experiencia de clase en relación con la estrategia metodología $A B P$, los recursos TIC y la unidad temática abordada; que se pudo evidenciar un reto interesante. En ocasiones complicado, pero algo práctico y diferente de aprender que ha de considerarse para seguir implementando. Adicionalmente, como parte de las observaciones realizadas durante las fases del $A B P$, se evidencia en los estudiantes una destacada respuesta de atención inicial, que dentro del aula se acompaña de actitudes de disposición, receptividad, seguimiento y participación orientados principalmente a las herramientas TIC como el weblog y el simulador, e igualmente en el tipo de proyecto a desarrollar. Es así que en conjunto estos comportamientos se convirtieron en una respuesta

actitudinal relevante en especial por su presencia estable aun cuando se desarrollan sesiones de trabajo de 1 a 2 horas, destacando la respuesta atencional frente a la presentación de las herramientas TIC de aquellos estudiantes que pudieron estar dispersos en algunos momentos.

En definitiva, se ha de resaltar la existencia de un interés situacional inicial en los estudiantes, considerado también como interés situacional activado que se evidencia a partir de las respuestas atencionales ante las situaciones presentadas: contenidos, actividades y estrategia $\mathrm{ABP}$ como se videncia a continuación: "Yo creo que ha sido algo muy interesante participar en el proyecto porque es algo diferente, es algo más práctico" Estudiante 38, " $A$ medida que avanzamos fue más interesante porque no es lo mismo que siempre... estar copiando, la misma práctica sino que fue algo más dinámico y me gustó" Estudiante 34.

En cuanto al Sentir por la clase, estrategias y estímulos, que evidencia las sensaciones, sentimientos, emociones, afectos y deseos de los estudiantes tanto al inicio como durante el proceso de aprendizaje aportando al conocimiento de la incidencia en la motivación académica de los estudiantes, es la reacción afectiva analizada a partir de los comportamientos y expresiones verbales, la que permite determinar disfrute y entusiasmo en la clase tanto desde las fases iniciales del $A B P$ como durante el proceso, principalmente durante el uso de los recursos TIC al interactuar de forma inmediata con los recursos tecnológicos asignados y participando del proceso investigativo.

Los estudiantes manifiestan que efectivamente les ha llamado la atención la clase y han disfrutado de ella en especial cuando se hacen las actividades prácticas, la experimentación, la simulación y el hecho de haber podido culminar el proyecto; disfrutan del trabajo en equipo y que de igual manera las TIC han sido algo divertido, innovador y de mucha utilidad para el proyecto. Sin embargo aparecen aspectos de valoración afectiva negativas con expresiones que evidenciaron momentos de desagrado, desanimo o preocupación hacia situaciones de la clase como la rúbrica de evaluación, las dificultades que podían enfrentar en el reto pero sobre todo el proceso de búsqueda inicial de información que al parecer no daba mayores resultados ante la cantidad y flujo de información en la red, adicionalmente el hecho de que se tenían escaso tiempo para dar cumplimiento con el proyecto.

Por otro lado, se evidencia también como parte del sentir por la clase, que los estudiantes valoran los contenidos, las estrategia ABP y las TIC utilizadas durante el aprendizaje en clase considerándolos significativos, importantes y útiles para su futuro. Lo anterior se evidencia con lo siguiente: "Me han parecido útil $y$ principalmente me ha gustado el simulador ya que nos ha ayudado en la parte del circuito" estudiante5, "Me parece que sigue siendo útil..., hacerlo nosotros. A mi parecer aprendemos más así" estudiante 53. Los estudiantes al valorar aspectos como la utilidad de los contenidos están considerándolos como parte de la cotidianidad en el que todo talento adquirido va a servir algún día, sin embargo se evidencia muy poca conexión personal a futuro: "Es útil pero a 
mí no me interesa para seguir con eso" Estudiante 6.

Es así que se valoran la autonomía y la puesta en práctica de estrategias autorreguladas para fortalecer el aprendizaje, en el que la mediación de los recursos TIC como el weblog, multimedia y simuladores fueron elementos importantes e innovadores durante la producción del proyecto y apropiación del aprendizaje.

No obstante, se evidencia que los estudiantes como parte del sentir por la clase, también manifestaron tensión emocional que en consecuencia desencadena la reacción afectiva positiva y negativa. La tensión se experimenta ante situaciones como el desconocimiento inicial de la temática, la posibilidad de un mal resultado en el proyecto y el enfrentamiento a una metodología que implica autorregulación de su propio aprendizaje.

Para finalizar, la implicación académica conductual se evidencia a partir de la persistencia e interacción social de los estudiantes en clase, siendo afectada en parte por distractores del aprendizaje, acompañada de igual forma por la reacción atencional, valoración afectiva $y$ cognitiva de parte de los estudiantes.

Es de resaltar que durante las observaciones analizadas, al inicio y durante el proceso, se mantiene la respuesta atencional y gusto por el trabajo, dando como resultado la participación de los estudiantes tanto en los procesos investigativos, como en el análisis, síntesis y elaboración de los proyectos, apoyados con recursos TIC multimedia, weblog de la clase, practicas con el simulador y recursos externos. Se evidencia una clara disposición y compromiso por la organización y avances en los proyectos, poniendo de manifiesto el desarrollo de su autonomía y generando un ambiente de trabajo ordenado, sereno y de relajación.

La persistencia se ha manifestado con el esfuerzo y constancia por las tareas académicas propuestas a pesar de las preocupaciones y distractores, que según los estudiantes es atribuido al interés por el tema, compañerismo, una adecuada estructura organizativa, compromiso de cada estudiante, posibilidad de práctica, ambiente escolar y los recursos ofrecidos, que les permitió hacer un trabajo constante con estados de concentración a pesar de los largos periodos de tiempo en algunas actividades.

Adicionalmente, la implicación académica también se ha evidenciado por medio de la estructura organizativa e interacción de cada equipo, donde cada integrante ha asumido un rol y una actividad dentro del trabajo, cooperando como grupo, y donde la posibilidad de trabajo en equipo les da la oportunidad de ayudarse, enriquecer las ideas y aprender de todos.

Avanzando en el razonamiento sobre la implicación académica conductual, se tratan los distractores del aprendizaje al identificar que es a través de ellos que se analizan las causas de ciertas conductas e interacciones negativas de algunos estudiantes que los alejaron de las actividades de aprendizaje. Es razonable encontrar que no es posible tener a la totalidad de un grupo de estudiantes en iguales estados de atención e implicación académica, más aun cuando existen factores externos reconocidos que desvían la atención de los estudiantes de las tareas escolares que para el caso de estudio como lo son: La tecnología (acceso a internet, celular), tiempo de actividades por más de 1 hora, acumulación de la jornada escolar, fallas en la conectividad, falta de equipos en algunas actividades. Sin embargo también atribuyen las distracciones al estrés durante la búsqueda de información y a la falta de estructura organizativa de ciertos grupos.

\section{Discusión}

A partir del análisis realizado en el tratamiento y análisis de los datos descrito en el ítem anterior, se determina que la implementación de la estrategia ABP mediada con herramientas TIC, puede incidir positivamente en la motivación académica de los estudiantes en la enseñanza de los conceptos básicos de los circuitos eléctricos. Lo anterior, evidenciado a través del interés de los estudiantes hacia las situaciones propuestas en el 
ambiente de aprendizaje, determinado por la presencia de respuestas atencionales, valoraciones afectivas positivas, valoración cognitiva a los contenidos, estrategia y recursos TIC y una adecuada implicación académica hacia el logro de los objetivos académicos.

\section{Conclusiones}

A lo largo del trabajo investigativo se logró demostrar que la implementación del ambiente de aprendizaje con la estrategia ABP mediada con herramientas TIC, con herramientas interactivas como el weblog y los simuladores, ha contribuido significativamente en la activación y desarrollo de emociones y actitudes positivas de los estudiantes, catalogadas estas como manifestaciones de interés que orientaron la conducta hacia el logro de las metas de aprendizaje, como lo fueron: trabajar competencias científicas y TIC a partir de la investigación. Lo anterior, permite concluir que, en el caso de estudio, hay una mejora en la motivación hacia el aprendizaje de los conceptos del área de tecnología.

Al mismo tiempo, se concluye que factores como las respuestas atencionales a los estímulos tanto en el inicio como durante el proceso, y las reacciones y valoraciones afectivas positivas, se orientan principalmente hacia los recursos TIC como tabletas, weblog, el simulador, juegos multimedia y chat, así como por el trabajo práctico y en equipo.

Aunque se experimenta cierta tensión emocional expresada como estrés académico, este se da en menor medida con respecto al evidenciado previamente a la intervención metodológica y puede ser un factor que siga presente cuando los estudiantes se vean enfrentados a ciertos niveles de dificultad y procesos que requieren de autorregulación, conocido como declive motivacional.

Otro de los factores asociados al interés evidenciado, es la valoración cognitiva hacia los contenidos, estrategia y recursos TIC utilizados, concluyendo que aunque hay valoración sobre la importancia a futuro por los contenidos abordados, los resultados no permiten definir si el interés situacional ha potenciado el interés personal por la temática. Lo anterior, en razón a que es un fenómeno que se establece a largo plazo e implica más exploración de parte de los estudiantes. Sin embargo, es posible establecer que aquellos estudiantes que manifestaron previamente tener un interés personal por el tema debido a su proyección profesional, han experimentado el interés situacional, y la estrategia metodológica ABP mediada con TIC, ha potenciado su interés personal, al considerarla como un elemento de valor cognitivo que desean seguir implementando.

En el caso del factor de implicación académica, evidenciado en los estudiantes durante las fases $\mathrm{V}$ y VI del proceso desarrollado en el ambiente de aprendizaje, reflejan el logro de una conexión significativa con el contenido, gracias a la mediación de las TIC y el planteamiento de estrategias activas de reto y elaboración de proyectos, que se revelan a través de conductas de persistencia durante la investigación, análisis, síntesis y elaboración de sus productos, mostrando también, la importancia de la organización e interacción social de trabajo en equipo. Es entonces, que a través de la implicación académica conductual se logra determinar que el interés por la clase y los contenidos se logra mantener.

Ahora bien, se reconoce la presencia de distractores del aprendizaje que afectaron de forma negativa la atención e implicación académica de algunos estudiantes, debido al tiempo de trabajo continuo por más de 1 hora, jornada escolar, fallas en la conectividad, falta de equipos, posibilidad de acceso a internet en juegos online, videos musicales y el celular; pero atribuido en parte al estrés generado en los estudiantes durante la investigación de los proyectos.

Según lo anterior, haber captado inicialmente la atención de los estudiantes desde la fase I de la estrategia $A B P$, valorando cognitiva $y$ afectivamente la propuesta de aprendizaje, al igual que se implicarán en las actividades académicas con una mínima afectación de los distractores del aprendizaje y el haber 
desarrollado proyectos funcionales sustentados conceptualmente, es un indicio claro de interés por el trabajo académico. Lo que implica una activación y orientación de la conducta, la fuerza, el deseo y energía hacia una meta académica que para el estudio se denominó motivación académica.

Finalmente es importante concluir que integrar las $T I C$ en un $A B P$, requiere tiempo en planear, estructurar, obtener habilidades y competencias docentes en IC, que permitan seleccionar $y$ diseñar material didáctico pertinente a las metas de aprendizaje. Donde el docente debe partir de su propio entusiasmo, hacerle comprender a los estudiantes porque es importante aprender y encontrar las estrategias adecuadas para obtener una óptima motivación.

\section{Referencias}

Amar, V. (2006). Planteamientos críticos de las nuevas tecnologías aplicadas a la educación en la sociedad de la información y de la comunicación. Pixel-Bit. Revista de Medios y Educación, enero 2006, núm. 27, Sevilla España. Universidad de Sevilla, pp. 79-87: recuperado el 7 de noviembre de 2016 de http://www.redalyc.org/articulo.oa?id=368027 06 ISSN 1133-8482.

Aristizábal, C. (2012). Aprendizaje Basado en Proyectos como estrategia de enseñanza y aprendizaje en la educación básica y media. Tesis de Maestría en la enseñanza de las Ciencias Exactas y Naturales. Universidad Nacional de Colombia. Recuperado el 7 de noviembre de 2016 http://www.bdigital.unal.edu.co/9212/1/43253 404.2013.pdf

Bisquerra A, R. (2009). Metodología de la investigación educativa ( $2^{\mathrm{a}}$ edición). Madrid. Editorial La Muralla S.A, pp 310 Recuperado de https://dialnet.unirioja.es/servlet/libro?codigo $=5826$

Boekaerts M. \& Boscolo P. (2002). Interest in learning, learning to be interested. Learning and Instruction, 12(4), pp 375-382.Recuperado de
https://linkinghub.elsevier.com/retrieve/pii/S09 5947520100007X

Colorado M, y Rodríguez. I. C. (2014). Proyecto de alfabetización científica y tecnológica: una propuesta e implementación en la enseñanza de las ciencias naturales para la educación básica primaria. Recuperado el 14 de mayo 2017 http://bibliotecadigital.univalle.edu.co/bitstrea m/10893/7191/1/3467-0430898.pdf

Corbin, J. y Strauss, A. (2002). Bases de la investigación cualitativa. Técnicas y procedimientos para desarrollar la teoría fundamentada ( $1^{a}$ ed.). Antioquia: Universidad de Antioquia. Recuperado de https://diversidadlocal.files.wordpress.com/201 2/09/bases-investigacion-cualitativa.pdf ISBN: 958-655-624-7 (volumen) ISBN: 958- 655-6239

Departamento de educación Universidades e Investigación del gobierno Vasco (2012). Competencia en el tratamiento de la información y competencia digital. Recuperado el 13 noviembre de 2016 de http://www.eskola20.euskadi.eus/c/document_l ibrary $/$ get_file?uuid $=5218 \mathrm{~d} 316-\mathrm{fa} 1 \mathrm{f}-49 \mathrm{~b} 4-$ 9eb3-e17f423298b0\&groupld $=408381$

Díaz S, L. (2011). La observación. Recuperado el 7 de marzo de 2017 de http://www.psicologia.unam.mx/documentos/p $\mathrm{df} /$ publicaciones/La_observacion_Lidia_Diaz_Sa njuan_Texto_Apoyo_Didactico_Metodo_Clinico_ 3_Sem.pdf

Domínguez, J. A., Carod, E.S. y Velilla, M.J. (2008). Comparativa entre el aprendizaje basado en proyectos y el aprendizaje basado en problemas. II jornadas de innovación docente, tecnologías de la información y de la comunicación e investigación educativa en la Universidad de Zaragoza 2008. Recuperado el 4 de diciembre 2016 de http://cmapspublic2.ihmc.us/rid=1J9HKH72N9B9GQG-

T9F/Informaci\%C3\%B3n\%20adjunta\%203.pdf 
Fros, F. (2013). La motivación y la emoción son dos caras de la misma moneda. Diálogo con Ross Buck, University of Connecticut. Recuperado el 11 de noviembre 2017 de http://www.homosentiens.com.ar/2013/dialog o-con-ross-buck/.

Fundación Enseña Chile (2015). Aprendizaje Basado En proyectos. Recuperado el (1 noviembre de 2016 de http://www.ensenachile.cl/wpcontent/uploads/2015/05/Aprendizajebasado-proyectos.pdf

Gómez G, Luz y Macedo B, Julio (2010). Importancia de las TIC en la educación básica Regular. Recuperado de http://revistasinvestigacion.unmsm.edu.pe/ind ex.php/educa/article/view/4776/3850

González, A., \& Paoloni, P. (2015). Implicación y rendimiento en Física: el papel de las estrategias docentes en el aula, y el interés personal y situacional del alumnado. Revista de Psicodidáctica, 20 (1), 25-45. Recuperado de http://www.redalyc.org/html/175/1753296800 3/

Gutiérrez, R. S., Duque, E. T., Chaparro, R. L., \& Rojas, Néstor R. (2018). Aprendizaje de los Conceptos Básicos de Realidad Aumentada por Medio del Juego Pokemon Go y sus Posibilidades como Herramienta de Mediación Educativa en Latinoamérica. Información tecnológica, 29(1), 49-58. https://dx.doi.org/10.4067/S071807642018000100049

Hernández S, R. (2014). Metodología de la investigación. México: Editorial Mc Graw Hill, pp 8-358. Recuperado de https://www.esup.edu.pe/descargas/dep_invest igacion/Metodologia\%20de\%20la\%20investiga ci\%C3\%B3n\%205ta\%20Edici\%C3\%B3n.pdf

Institución Educativa San Sebastián (2015). Proyecto Educativo Institucional (PEI). Colombia, La plata, Huila. pp 22.

Maldonado P, M. (2008). "Aprendizaje Basado en Proyectos. Una experiencia en educación".
Revista Laurus, Año 14, No 28. Venezuela. Universidad Pedagógica Experimental, pp 158180. Recuperado el 25 de febrero 2017 de http://www.redalyc.org/pdf/761/76111716009. pdf

Ministerio de Educación Nacional MEN (2008). Orientaciones generales para la educación en tecnología. Ser competente en tecnología: juna necesidad para el desarrollo! Bogotá, Colombia. Imprenta Nacional, pp 3. Recuperado de https://www.mineducacion.gov.co/1621/article s-160915_archivo_pdf.pdf

Núñez, J. (2009). Motivación, aprendizaje y rendimiento académico. Recuperado de http://www.educacion.udc.es/grupos/gipdae/d ocumentos/congreso/xcongreso/pdfs/cc/cc3.p $\mathrm{df}$

Ospina M, C. (2013). Las TIC como herramienta de motivación en el aula. Tesis de Maestría en Informática Educativa. Universidad de la Sabana, Bogotá. Recuperado de https://intellectum.unisabana.edu.co/bitstream /handle/10818/5358/129394.pdf?sequ\}

Renninger K, A. (2009). Desarrollo de interés e identidad en la instrucción: un modelo inductivo, psicólogo educativo, 44: 2, 105-118, DOI: 10.1080 / 00461520902832392

Stake, Robert (1999). Investigación con estudio de casos ( $2^{a}$ edición). España. Ediciones Morata S. L, pp 12-17. Recuperado de https://www.uv.mx/rmipe/files/2017/02/Investi gacion-con-estudios-de-caso.pdf

Taylor, S.J. y Bogdan, R (2002). Introducción a los métodos cualitativos de investigación. España. Editorial PAIDÓS, pp 21. Recuperado de https://iessb.files.wordpress.com/2015/07/05_t aylor_mc3a9todos.pdf 